\title{
The New Remote Alarm System for Household Door and Window Intrusion Based on ZigBee
}

\author{
Yulong $\mathrm{HaO}^{1, \mathrm{a}}$, Jiaoyu $\mathrm{Liu}^{2, \mathrm{~b}}$ and Qiang Wen ${ }^{2, \mathrm{c}}$ \\ 1. School of Automation Wuhan University of Technology Wuhan,430070,China \\ 2. School of Automation Wuhan University of Technology Wuhan,430070,China \\ a.whuthyl@163.com, b.whut_Liujiaoyu@163.com, c.whut_wenqiang@163.com
}

Keywords: ZigBee; remote alarm system; magnetic sensor; wireless communication.

\begin{abstract}
In view of high-cost and low real-time of traditional alarm system for home intrusion ,this paper proposes and implements the one new remote alarm system for household doors and windows intrusion based on ZigBee and WIFI, GPRS model , this system uses magnetic sensor on household doors and windows to detects the state of doors and windows for real-time, and uses wireless communication technology to send the alarm information produced by magnetic sensor when the door or the window is opened to the mobile phone, PC monitor, and start the alarm system, it has realized the wireless remote monitoring and alarm function, so that whether at home, or the outer ,the householder can real-time monitor the security situation about the home and can take appropriate security measures. Because it is low-cost and it can be easy to be achieved, this system has a high practical value.
\end{abstract}

\section{Introduction}

With the development of science and technology, both in industry and agriculture, or home network, the demand for wireless access technology is in increasing, nowadays the development tendency of the wireless communication and wireless network are presenting index increased. Compared to the traditional alarm system for home intrusion, this design uses the ZigBee wireless technology and router port mapping technology, and has completed communication in inside network and in outside network .There are following advantages: low power consumption, low cost and high reliability, it provides a perfect solution to alarm system needing high real-time capability, difficult to damage, easier to achieve. Anytime and anywhere, householder can know the security situation of doors and Windows in the home through the phone monitoring terminal or PC monitoring terminal.

\section{The System Function Introduction}

As shown in figure 1, this system includes terminal node, data transceiver module, alarm module, mobile monitoring terminal and PC monitoring terminal. The terminal node is composed of magnetic sensor and ZigBee EndDevice, and data transceiver module is composed of ZigBee Coordinator and WIFI module. The state of the doors and Windows can be detected by magnetic sensor in real time, if the door or window is opened, magnetic sensor will produce alarm signal, then ZigBee terminal node will collect the alarm information, and send to the ZigBee coordinator by wireless transmission. At this time, on the one hand, Coordinator starts the acousto-optic alarm system, on the other hand, it will send the alarm information to the wireless router via a serial port turning into WIFI. Wireless routers can send the data via the local area network(LAN) to mobile monitoring terminal and PC monitoring terminal in LAN, can also through the router port mapping function to access outside network to send data to mobile phone monitoring terminal in outside network. Finally the householder will get the warning information timely, and take the corresponding security measures in due course. The householder can also query the state of doors and windows by the monitoring. 

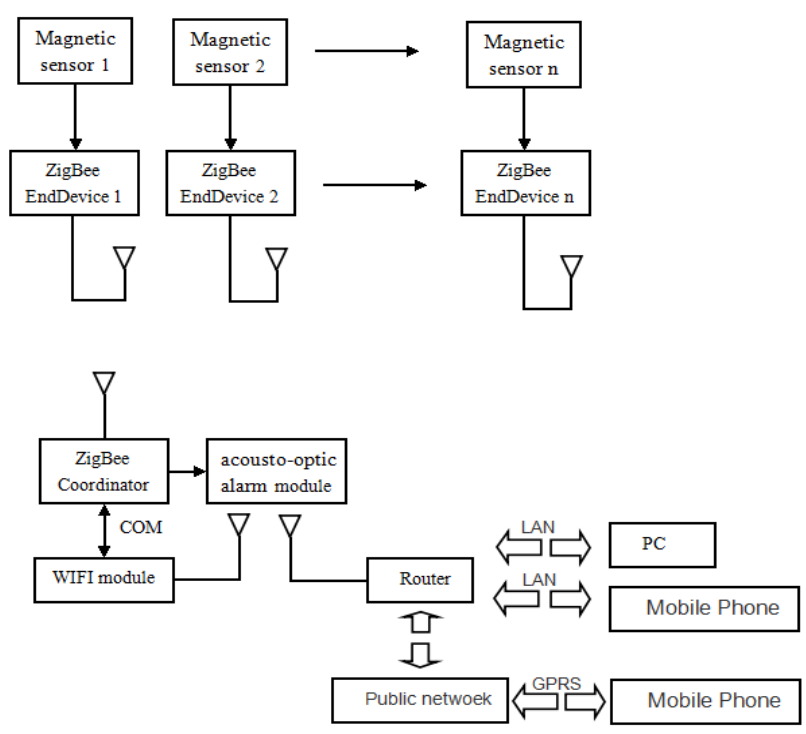

Fig. 1 System structure diagram

Pictured below is the system working condition display figure. Figure 2(a) shows the query result within LAN, when we send "C"(CHECK) to WIFI module ; Figure 2(b) shows the alarm information within GPRS. When the doors and Windows are in a safe state, monitoring terminal will get the clues of "Security", and when the doors or windows are open, monitoring terminal can show "Warning" alarm the clues unremittingly, and Coordinator will start alarm system in the home.

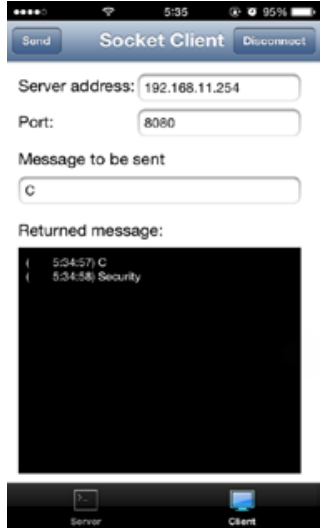

(a)

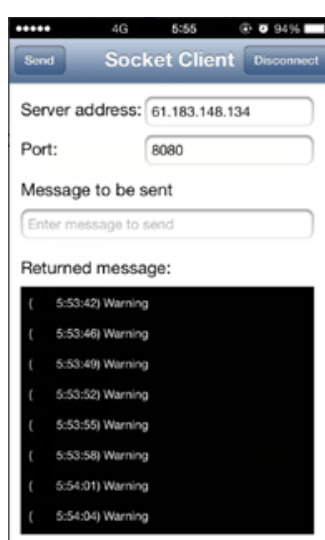

(b)

Fig.2 System working condition

\section{System Hardware Introduction}

\subsection{ZigBee wireless transceiver devices}

In this paper the core chip of ZigBee devices is CC2530 launched by TI company,CC2530 fully support ZigBee2007 protocol not only, and still combines the leading the good performance of RF transceiver and industry standards enhanced 8051 CPU.

CC2530 is a true System on Chip(SoC) used in application of the 2.4 GHz IEEE 802.15.4, ZigBee and RF4CE. It can establish a powerful network nodes by using very low cost of materials and also can build in a variety of network protocols , including ZigBee protocol stack. There are four different flash version of it:CC2530F32/64/128/256, respectively with 32/64/128/256 KB flash memory.CC2530 have different operation mode, making it especially adapted to ultra low power requirements of the system. Short time in running mode conversion further ensure that the low energy consumption.

\subsection{WIFI module}

In this system ,the WIFI module is ATK-RMO4 ,it is a high-performance UART-ETH - WIFI (serial port - Ethernet and wireless networks) module launched by ALIENTEK. 
ATK-RMO4 uses a serial port communication with MCU, built-in TCP/IP protocol stack, and it can realize serial port, Ethernet and wireless network transformation between the three interfaces.

ATK - RM04 module function can be divided into four modes: the default mode (router mode), a serial port turn-into WIFI STA mode (COM - WIFI STA), a serial port turn-into WIFI AP (COM WIFI AP), a serial port turn-into Ethernet (COM - ETH). It takes advantage of the function of turning serial port into the wireless network, turns the traditional way of serial port to read and write ZigBee coordinator data into to read and write ZigBee coordinator data by wireless network. Namely, set ATK - RM04 to a serial port turn-into WIFI STA mode, then let it connect to the wireless routers, and become a WIFI local area net equipment.

\subsection{Magnetic sensor module}

Magnetic sensor can be divided into cable magnetic sensor and wireless magnetic sensor. Cable magnetic is embedded installation, it has the advantage of high concealment, and is used for wood or aluminum alloy doors and Windows, so this system takes the cable magnetic sensor, let it connect to ZigBee EndDevice to collect the state information of doors and windows, then the information will be sent to Coordinator by ZigBee network.

Magnetic sensor has two parts: the permanent magnet and reed switch. They are respectively installed on the door (window), and on the door (window) frame, when permanent magnet close to reed switch, the lines of magnetic force makes reed switch off contact, at this time, circuit disconnect; When the door(window) is opened, reed switch and permanent magnet distance becomes far, at this time, reed switch contact closure because of the action of no magnetic field lines connect circuits, and generate alarm information. In this way, door switch state can be effectively detected .

\section{The System Software Introduction}

\subsection{ZigBee technology introduction}

In the network, in order to complete communication, must use multilayer on multiple protocols. These protocols in hierarchical order together, constitute a protocol stack. Protocol stack is refers to the network layer protocol in the sum of a set of protocol specification. It vividly reflects a network file transfer process: by the upper level protocol to the underlying, again by the underlying protocol to the upper.

ZigBee technology is a new kind of short distance, low rate of wireless network technology, and it is based on IEEE 802.15.4, this is the IEEE wireless personal area network PAN (Personal Area Network) working group of a standard. IEEE 802.15.4 processing only low-level MAC layer and physical layer protocols, so the ZigBee technology alliance had standardized the network layer protocol and API of IEEE802.15.4,and was responsible high-level application of ZigBee technology and marketing work, and finally formed the ZigBee protocol stack(Z-stack). ZigBee technology has powerful networking function, it supports the star structure, the structure of the mesh (Mesh) and cluster structure (Cluster tree) three main types of self-organized wireless network. The Mesh, in particular, has a strong network robustness and reliability. ZigBee adopted CSMA-CA collision avoidance mechanism, and use the Direct Sequence Spread Spectrum technology, there are three spectrum can be used, respectively are 2.4 GHZ ISM Spectrum,868 MHZ Spectrum of Europe and the United States of 915MHZ Spectrum..ZigBee transmission rate between 20 to $250 \mathrm{Kbps}$, with low rate, low power consumption, high number of links, long transmission distance, etc. Therefore, ZigBee technology can be applied to induction network (Sensor Network), has a considerable advantage.

$\mathrm{Z}$ - stack is also starting from the main () function, the main() function to perform two tasks: one is the initialization of the system, and the second is starting the round robin system (OSAL) operation. Figure 3 is OSAL work flow chart. 


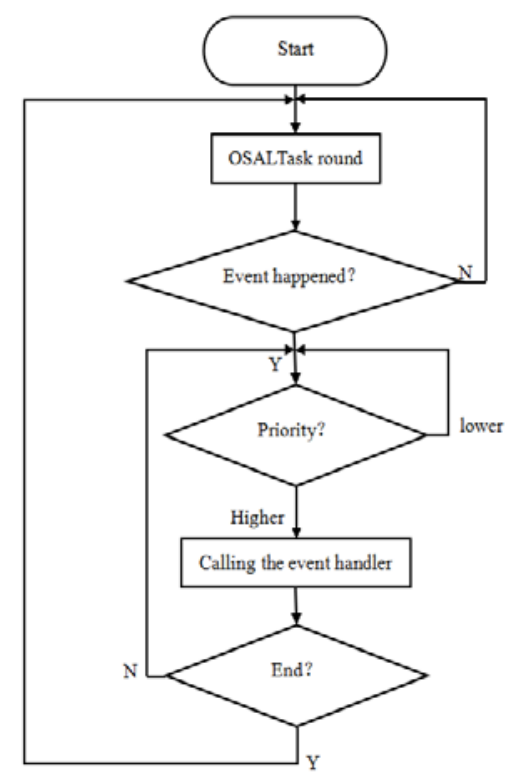

Fig.3 OSAL Work flow chart

Considering the network space of the system structures, the cost of system structures and the recovery of function of ZigBee network when network is broken , the system of network uses a star network. The topology structure need to be a coordinator and a number of terminal equipment (determined by the number of doors and windows), The definition of ZigBee device and the application layer data acquisition program of Z-stack are completed in Embedded Workbench IAR development platform.

\subsection{Port mapping technology introduction}

Port mapping technology is mapping a port of outside network to a machine of inside network, and provide the corresponding services. In this way ,router can map a port to ATK-RM04,then set ATK RM04 to TCP server, and set the phone to TCP client. When the householder accesses to the network IP router, the access requests will be transferred to ATK - RM04.Finally,the householder in outside can query the safety status of home.

\section{Conclusion}

This paper take full advantage of the ZigBee technology of low power consumption, low cost, easy realize and high reliability etc, puts forward and implements the new type of family security alarm system, absorbing the advantages of the traditional alarm system for family security, adding the new function design and improvement to make the system that in can be widely used in every family. The working effect of the system has reached expectations, because of stability and real-time performance, this design has high use value and application prospect.

\section{References}

[1]. Ting Jiang, Cheng-lin Zhao. ZigBee purple bee technology and application [M]. Beijing: people's university of posts and telecommunications publishing house, 2006.

[2]. Wen-zhong Li ,Chao-yu Duan. ZigBee wireless network technology introduction and practice [M]. Beijing: Beijing university of aeronautics and astronautics press, 2007.

[3]. Hao-qiang Tan. C language program design [M]. Beijing: Tsinghua university press, 2006.

[4]. TexasInstruments.CC2530Datasheet[EB/OL].2009[2010-05-03]. http://www.ti.com.cn/Product/cn/cc2530\#technicaldocunments

[5]. Xiao-qiang Wang ,Jun-ou Yang, Ning-lin Huang. ZigBee wireless sensor network design and implementation [M]. Beijing: chemical industrypress,2012.5. 